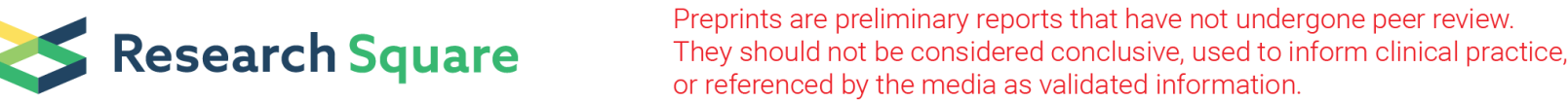

\section{Platelet Priming Following Naturally Occurring Thermal Burn Injuries And Wildfire Smoke Exposure is Associated with Intracardiac Thrombosis in Feline Survivors}

Ronald H. L. Li ( $\square$ rhli@ucdavis.edu )

University of California, Davis

Avalene W. K. Tan

University of California, Davis

Yu Ueda

North Carolina State University

Joshua A. Stern

University of California, Davis

Mehrab Hussain

University of California, Davis

Satoshi Haginoya

University of California, Davis

Ashley N. Sharpe

University of California, Davis

Catherine T. Gunther-Harrington

University of California, Davis

Steven E. Epstein

University of California, Davis

Nghi Nguyen

University of California, Davis

\section{Research Article}

Keywords: Platelet priming , wildfire smoke exposure, thermal burn injuries, feline survivors

Posted Date: August 6th, 2021

DOI: https://doi.org/10.21203/rs.3.rs-778724/v1

License: (c) (1) This work is licensed under a Creative Commons Attribution 4.0 International License.

Read Full License 
Page 2/24 


\section{Abstract}

Wildfires pose a major health risk for humans, wildlife, and domestic animals. We previously discovered pathophysiologic parallels between domestic cats with naturally occurring smoke inhalation and thermal burn injuries and human beings with similar injuries; these were characterized by transient myocardial thickening, cardiac troponin I elevation and formation of intracardiac thrombosis. While the underlying mechanisms remain unclear, results from murine models suggest that platelet priming and activation may contribute to a global hypercoagulable state and thrombosis. Herein, we evaluated and compared the degree of platelet activation, platelet response to physiologic agonists and levels of platelet-derived microvesicles (PDMV) in 29 cats with naturally occurring wildfire thermal injuries, 21 clinically healthy cats with subclinical hypertrophic cardiomyopathy (HCM) and 11 healthy cats without $\mathrm{HCM}$ (CC). We also quantified and compared circulating PDMVs in WF cats to CC cats. In addition, we examined the association between thrombotic events, severity of burn injuries, myocardial changes, and the degree of platelet activation in cats exposed to wildfires. Flow cytometric detection of platelet surface P-selectin expression showed that cats in the wildfire group (WF) had increased platelet response to adenosine diphosphate (ADP) and thrombin compared to the 2 control groups indicating the presence of primed platelets in circulation. In addition, cats in the WF group had increased circulating levels of PDMV, characterized by increased phosphatidylserine on the external leaflet. Cats in the WF group with documented intracardiac thrombosis had elevated platelet activation and platelet priming in the presence of ADP or thrombin. While high dose arachidonic acid (AA) mostly resulted in platelet inhibition, persistent platelet activation in response to AA was noted among cats in the WF group with intracardiac thrombosis. Univariate and multiple logistic regression analyses demonstrated that increased platelet response to AA was independently associated with thrombotic events. This is the first study reporting the significant association between platelet priming and intracardiac thrombosis in domestic cats with naturally occurring wildfire-related injuries and smoke inhalation. Further studies are required to delineate additional mechanisms between inflammation and thrombosis, especially regarding platelet primers and the cyclooxygenase pathway.

\section{One Sentence Summary}

Platelet activation and shedding of platelet-derived microvesicles due to platelet priming is present following naturally occurring wildfire smoke exposure and thermal burn injuries in a population of domestic cats.

\section{Introduction}

The most recent wildfires in Northern California (2018 California Camp Fire, 2020 Californian Fires), Brazil (Amazon Fires 2019) and Australia (2019/2020 Australian bushfires) have been the most destructive in modern history, injuring thousands and claiming the lives of millions of animals. As the intensity and frequency of wildfire disasters increase due to climate change, wildfires pose a major health risk for humans, wildlife and domestic animals. An observational study in domestic cats during 2 recent periods 
of Californian wildfires (Tubbs Fire 2017, Camp Fire 2018) documented the clinical impacts of wildfire injuries on the cardiovascular system ${ }^{1}$. Echocardiography not only documented the presence of transient myocardial thickening (MT) but also spontaneous echocardiographic contrast and intracardiac thrombosis (SEC $\pm T$ ), suggestive of a hypercoagulable state following wildfire exposure. While human studies have also demonstrated positive associations between wildfire-related injuries like smoke inhalation and thermal burns and increased risk of cardiovascular events, such as myocardial infarction and ischemic diseases ${ }^{2-5}$, the underlying mechanisms of these observations are not well understood.

People and animals rescued from wildfires often succumb to thermal burn injuries and smoke inhalation, both of which can result in severe derangements in hemostasis. Observational studies in humans and murine models of burn injuries describe an initial hypercoagulable state despite the presence of thrombocytopenia ${ }^{6-9}$. This finding suggests that systemic platelet activation, which likely precedes platelet consumption, plays a crucial role in mediating hypercoagulability as a result of burn injuries ${ }^{7,10,11}$. Although the exact mechanisms of increased platelet activation in these patients are unknown, endothelial stress and injury during inflammation may facilitate platelet rolling and activation, and subsequent platelet-immune cell interactions via toll-like receptors (TLR) fueling further inflammation ${ }^{12-15}$. This highlights the important role that platelets play in linking inflammation with the coagulation system. Platelet activation also triggers the shedding of PDMV. As mediators of hemostasis and inflammation, overzealous amounts of circulating PDMV may further aggravate an already hypercoagulable state ${ }^{11,13,16}$. Inhalation of ambient particulate matter and noxious gases generated during wildfires may also directly activate circulating platelets. In vitro and in vivo animal models demonstrate that inhalation of particulate matter can initiate platelet priming, an exaggerated response to physiologic agonists, resulting in systemic platelet activation and elevation of circulating PDMV. ${ }^{17}$ To date, cardiovascular events and thrombosis as a result of increased platelet priming and shedding of PDMV in animals with naturally occurring wildfireinjuries and smoke inhalation have never been demonstrated.

Cats with hypertrophic cardiomyopathy ( $\mathrm{HCM})$, the most common primary cardiomyopathy, are at high risk of thromboembolic complications such as sudden death and arterial thromboembolism. Although the exact mechanisms of thrombosis secondary to HCM are unclear, increased platelet priming, endothelial dysfunction and blood flow stasis due to left atrial (LA) dysfunction are proposed to play a key role in contributing to a hypercoagulable state ${ }^{18-20}$. Since cats with wildfire-related injuries were previously shown to have transient cardiomyopathies, it is plausible that cardiovascular complications and thrombosis noted in these cats may be secondary to similar pathophysiologic mechanisms in HCM cats. A better understanding of the mechanisms behind WF-related thrombosis will aid in developing more effective thromboprophylaxis and antithrombotic therapies in animals with naturally occurring WF injuries.

To address this knowledge gap, we evaluated and compared the degree of platelet activation and platelet ex vivo response to physiologic agonists in cats with naturally occurring WF injuries, and clinically 
healthy cats with or without subclinical HCM. Circulating PDMVs between and healthy controls cats and cats with wildfire-related injuries were also quantified and compared. Through this observational study, we examined the association between thrombotic events, severity of burn injuries, myocardial changes, and the degree of platelet activation and priming in cats exposed to WF. We hypothesized that cats with WF injuries, similar to HCM cats, would have increased response to physiologic agonists due to platelet priming. In addition, this potentiated platelet response to agonists would be associated with thrombotic events.

\section{Results}

\section{Study population}

Twenty-nine cats were presented to the University of California, Davis William R. Pritchard Veterinary Medical Teaching Hospital for evaluation and treatment in 2018 for Camp Fire wildfire related injuries. From a separate population of client-owned cats, twenty-one cats with asymptomatic subclinical HCM and 11 healthy cats without HCM were enrolled in the HCM and Control Cat (CC) groups, respectively. Of the 29 WF cats, 16 were males and 13 were females. In the HCM group, 14 were males and 7 were females. In CC group, 8 were males and 3 were females. No significant differences in the proportion of males and females were found between either HCM or CC groups when compared to WF cats $(p=0.56, p$ $=0.47$, respectively). Less WF cats were castrated or spayed (18/29) compared to the HCM $(21 / 21, p=$ $0.0014)$ and $C C$ groups $(11 / 11, p=0.02)$. Cats in the WF group also weighed less $(4.0 \mathrm{~kg}, 3.7-4.5)$ than those in the $\mathrm{HCM}(5.8 \mathrm{~kg} \pm 1.6, \mathrm{p}<0.001)$ and $\mathrm{CC}(5.5 \mathrm{~kg} \pm 1.0, \mathrm{p}=0.0010)$ groups.

Following enrollment, a complete blood count was performed in all cats. Neutrophil count was significantly different among the 3 groups $(p=0.0012)$. Cats in the WF group had higher neutrophil counts compared to cats in the HCM group $(p=0.00060)$ (Table 1). However, there were no significant differences in platelet count or mean platelet volume among the 3 study groups $(p=0.92, p=0.23$, respectively). 
Table 1

Patient characteristics and complete blood count parameters of each study group. WF = Wildfire cats, $\mathrm{HCM}=$ Healthy cats with hypertrophic cardiomyopathy and $\mathrm{CC}=$ Healthy cats without hypertrophic cardiomyopathy. IVSd = Interventricular septal thickness at diastole, LVPWd = Left ventricular posterior wall thickness at end-diastole, IVS = Interventricular septum, LVPW = Left ventricular posterior wall.
Study groups
WF $(\mathrm{N}=$
$\mathrm{HCM}(\mathrm{N}=$
21)
$\mathrm{CC}(\mathrm{N}=11) \quad P$ value
WF vs WF vs HCM

Complete blood count parameters

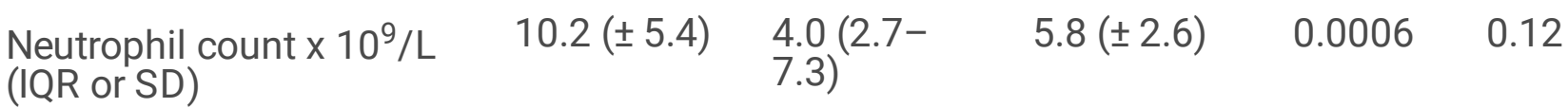

$\begin{array}{llllll}\text { Platelet count } x 10^{9} / \mathrm{L} & 204( \pm 87) & 234( \pm 87) & 208( \pm 106) & 0.23 & 0.89\end{array}$

(IQR or SD)

$\begin{array}{llllll}\text { Mean platelet volume, fl } & 10.8 & 11.8 & 10.7( \pm 1.7) & 0.08 & 0.48 \\ \text { (IQR or SD) } & (10.3- & (10.6- & & & \end{array}$

Echocardiographic data

2D IVSd, mm

$4.8( \pm 1.0)$

$6.7( \pm 1.2)$

$4.8( \pm 0.5)$

$<$

$0.98<$

(IQR or SD)

2D LVPWd, mm

$5.2( \pm 0.8) \quad 6.3( \pm 1.2)$

$4.9( \pm 0.5)$

0.0009

0.71

0.0011

(IQR or SD)

$\mathrm{M}$ mode IVS, $\mathrm{mm}$

$4.7( \pm 0.9)$

$5.6(4.9-$

$6.2)$

(IQR or SD)

$\mathrm{M}$ mode LVPW, $\mathrm{mm}$

$5.0( \pm 0.9)$

$5.8( \pm 0.8)$

$4.5( \pm 0.6)$

0.0075

0.18

0.0004

(IQR or SD)

$\begin{array}{lllllll}\text { Left atrial: Aorta } & 1.4(1.3- & 1.5(1.3- & 1.4( \pm 0.1) & >0.99 & >0.99 & >0.99\end{array}$

(IQR or SD)

$\begin{array}{lllllll}\text { Left auricular velocity, } & 59.2( \pm & 54.4 & 43.4 & >0.99 & 0.048 & 0.14 \\ \mathrm{~cm} / \mathrm{s} & 14.7) & (48.2- & (39.3- & & & \\ & & 61.0) & 52.5) & & \end{array}$

(IQR or SD)

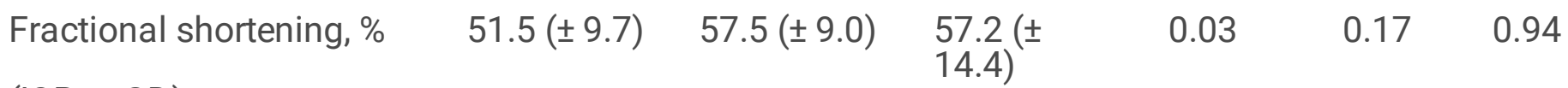
(IQR or SD) 
Of the 29 cats in the WF group, 26 (89.7\%) survived to hospital discharge. Of the 3 cats that did not survive, all had a burn severity score of 3 . One (3.4\%) cat died of sudden death. Necropsy and histopathology of the heart and lungs showed left ventricular (LV) concentric hypertrophy and severe multifocal histiocytosis with intracellular pigment accumulation consistent with smoke inhalation. Two (6.9\%) cats were euthanized; one cat was euthanized due to recurrence of congestive heart failure (CHF) and one cat was euthanized due to deterioration of respiratory signs unrelated to cardiac causes. Thoracic radiographs of this cat showed diffuse bronchointerstitial pattern without cardiomegaly.

\section{Echocardiographic findings}

Nine $(9 / 29,32.1 \%)$ cats in the WF group had evidence of myocardial thickening (MT). Expectedly, all HCM cats had significant MT in all 4 echocardiographic measurements compared to cats in the WF and CC groups $(p<0.01)$. No significant differences in these measurements were found between the WF and CC groups (Table 1). Of the 29 WF cats, $6(20.7 \%)$ cats had evidence of LA enlargement compared to $6 / 21$ $(28.6 \%)$ cats in the HCM group ( $p=0.74)$. None of the cats in the CC group had documented LA enlargement. Left auricular flow velocity (LAuV) was higher in WF group compared to CC group $(p=$ 0.048 ) but was not different from that in HCM cats ( $p>0.99)$. Only 1 WF cat had documented pericardial effusion. Spontaneous echocardiographic contrast with or without organized thrombus (SEC $\pm T$ ) was found in $13 / 29$ cats (44.8\%) in the WF group. Additional echocardiographic parameters are summarized in Table 1.

\section{Cats exposed to wildfires had evidence of platelet priming}

To assess and compare the degree of platelet activation in response to platelet agonists, P-selectin on platelets was labeled with fluorochrome-conjugated P-selectin antibody and measured by flow cytometry before and after ex vivo treatment with adenosine diphosphate (ADP) or thrombin. Although we did not detect any differences in platelet activation in circulating platelets among the 3 study groups $(p=0.26)$ (Fig. 2A), there was evidence of platelet priming given that platelets in the WF group had increased responses to ADP and thrombin (Fig. 1B, Fig. 2B, C). When assessing the level of P-selectin expression, measured as median fluorescence intensity $\left(\mathrm{MFI}, \log _{10}\right.$ scale), WF cats had the highest response to $A D P$ among the 3 groups $(p=0.0002)$ with WF cats (MFI = 3718; IQR: 2866-4517) being higher than HCM (MFI = 2432; IQR: 1707-3168; $p=0.00020)$ and CC cats (MFI = 2215; IQR 1772-2554, $p=0.0010)$. No difference was found between HCM and CC cats $(p=0.96)$ (Fig. 1B). ADP did not result in significant changes in the number of P-selectin positive platelets among the 3 groups $(p=0.47)$.

Thrombin stimulation resulted in a significant difference in the percentage of P-selectin positive platelets among the 3 groups $(p=0.00040)$ with WF group $(64.10 \%$; IQR $25.40-74.77)$ being significantly higher than HCM (37.30\%; IQR 45.05-85.50, p = 0.0018) and CC cats (13.10\%; IQR 10.60-27.20, p = 0.00020). Platelets from cats with HCM had a greater response to thrombin than CC cats ( $p=0.014)$ (Fig. $2 C$ ).

\section{Arachidonic acid (AA) led to platelet inhibition in cats exposed to wildfires}


We then further characterized ex vivo platelet activation in response to $1 \mu \mathrm{M} A \mathrm{~A}$ in the WF group. Unexpectedly, AA led to platelet inhibition (Fig. 1A) as indicated by a significant decrease in P-selectin positive platelets compared to resting platelets (3.87\%; IQR 0.41-32.4 vs. 63.8\%; IQR 28.88-71.44; $\mathrm{p}<$ 0.0001 ) (Fig. 3A). However, this did not alter P-selectin MFI (MFI = 1341; IQR 620.81-1841 vs. MFI = 1696; IQR 970.50-2265, $p=0.30$ ) (Fig. 3B).

\section{Cats with intracardiac thrombosis had increased platelet activation and platelet priming}

Cats in the WF group with SEC $\pm \mathrm{T}$ had a higher percentage of P-selectin positive platelets than those without SEC \pm T (65.8\%; IQR 52.65-70.95 vs. $26.2 \%$; IQR 16.80-70.55, $p=0.049$ ) (Fig. 4A). Platelet priming was also evident in WF cats with SEC $\pm T$ as demonstrated by a higher percentage of P-selectin positive platelets after stimulation with $\operatorname{ADP}(70.33 \% \pm 8.64$ vs. $56.55 \% \pm 19.40, p=0.030)$ and thrombin $(60.98 \% \pm 19.97$ vs. $39.93 \% \pm 28.57, p=0.036)$ compared to those without SEC $\pm T$ (Fig. $4 \mathrm{C}$ and $4 \mathrm{E})$. Of the 3 platelet agonists, only AA was found to significantly upregulate surface P-selectin MFI in cats with SEC $\pm T$ (Fig. $4 \mathrm{H}$ ). Cats with SEC $\pm T$ had persistent platelet activation in the presence of $A A$ with an increased MFI (1574; IQR 1184-3325) compared to those without ICT (760; IQR 517-1585, $p=0.040)$. No differences in P-selectin MFI were detected in resting, ADP- or thrombin-treated platelets $(p=0.18,0.30$, 0.47 , respectively) from WF cats with or without $S E C \pm T$.

\section{Cats exposed to wildfires had elevated circulating platelet- derived microvesicles compared to healthy controls}

We next measured the concentration (number/ $\mu \mathrm{L}$ ) of PDMV and number of PDMV positive for phosphatidylserine (PS) in platelet poor plasma by flow cytometry. PDMV were identified by size, scatter properties, as well as, platelet integrin subunit, $\beta 3$ (CD61), and annexin V (Fig. 5A). No platelet agonists were added to the samples prior to PDMV analysis. Scatter profile and PDMV numbers from platelets treated with calcium and A23817 were similar to those seen in cats from the WF group (Fig. 5B,C). We found that WF cats had significantly elevated concentrations of circulating PDMV compared to CC cats

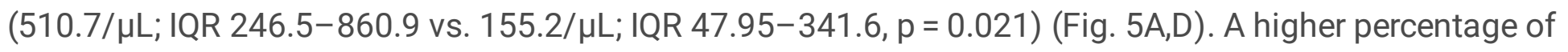
PDMV consisted of PS on the outer leaflet (\% positive) was found in WF cats compared to CC cats $(65.39 \% \pm 23.78$ vs. $42.46 \% \pm 25.90, p=0.024)$ (Fig. $5 E)$. Neither the concentration of PMV $(514.5 / \mu \mathrm{L}$; IQR

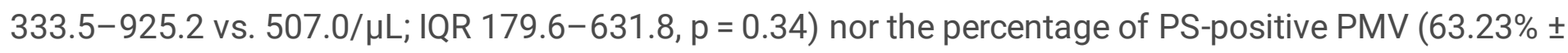
25.62 vs. $67.25 \% \pm 22.79, p=0.50$ ) were found to be different among WF cats with and without SEC \pm T.

\section{Echocardiographic changes had no impact on platelet priming, spontaneous echo contrast or intracardiac thrombosis in cats exposed to wildfires}

Since cats with subclinical HCM have previously been reported to have increased platelet activation and increased platelet priming, we sought to assess the effects of myocardial changes on platelet activation 
and the formation SEC $\pm T$ within the WF group. ${ }^{19,20}$ Cats with SEC $\pm T$ did not have significant differences in LV wall thickness $(5.36 \mathrm{~mm} \pm 0.89$ vs. $5.67 \mathrm{~mm} \pm 0.88, p=0.41)$ or LAuV $(59.79 \mathrm{~cm} / \mathrm{s} \pm 14.51$ vs. $58.66 \mathrm{~cm} / \mathrm{s} \pm 15.31, p=0.85)$ compared to those without $S E C \pm T$. We also did not find significant differences in platelet activation and response to platelet agonists in those with documented MT. Pselectin in resting platelets were not significant different between cats with and without MT (MFI:1623; IQR 1053-4000 vs. MFI:1699; IQR 862.8-2171, $p=0.35$ ). Similarly, response to ADP (MFI: $4077 \pm 1342$ vs. MFI: $3506 \pm 1128, p=0.29$ ), thrombin (MFI: $2414 \pm 1961$ vs. MFl:1939 $\pm 954.8, p=0.51$ ) and AA (MFl: 1077; IQR 742-1422 vs. MFI:1558; IQR 578-1922, $p=0.39$ ) were comparable between the 2 groups. There were also no differences in P-selectin expression among WF cats with normal LAuV $(>47 \mathrm{~cm} / \mathrm{s})$ compared to those with low LAuV (<47cm/s) in resting (MFI:1673; IQR 996-2282 vs. MFI: 1808; IQR 835-2779, p = 0.81), ADP- (MFI:3835; IQR 2832-4551 vs MFI: 3004; IQR 2510-3922, p = 0.32) thrombin(MFI: 1713; IQR 1014-2273 vs. MFI: 2477; IQR 1329-3196, p = 0.48) or AA- (MFI: 1238; IQR 562.8-1841 vs. MFI: 1664; IQR 872.5-1947, $p=0.43$ ) activated platelets. Similar findings were noted in P-selectin positive platelets.

\section{Platelet activation in response to arachidonic acid (AA) is associated with intracardiac thrombosis in cats with naturally occurring wildfire injuries}

To determine the association between platelet activation, severity of burn injuries and intracardiac thrombosis $(S E C \pm T$ ), univariate and multiple logistic regression analyses were performed. We found a significant association between SEC $\pm T$ and platelet activation in response to $A A(p=0.0066)$. However, no association between SEC $\pm T$ and severity of burn injury $(p=0.21)$ was found. Other variables such as resting P-selectin expression, response to $A D P$, measured as $\log _{10} \mathrm{MFI}$ fold change, and neutrophil count were not associated with SEC $\pm T(p>0.5)$. Simple logistic regression analysis revealed that the magnitude of platelet response to AA ( $\log _{10} \mathrm{MFI}$ fold change) predicted clot formation (coefficient 3.88, $95 \% \mathrm{Cl} 0.27-7.48, \mathrm{p}=0.0022$ ). Further analysis by multiple logistic regression to predict $\mathrm{SEC} \pm \mathrm{T}$ based on severity of burn injuries, resting P-selectin expression (MFI), magnitude of platelet response to AA or ADP ( $\log _{10}$ MFI fold change), and neutrophil count was performed. Platelet response to AA remained to predict formation of SEC $\pm \mathrm{T}$ (coefficient $3.77,95 \% \mathrm{Cl} 0.19-7.31, \mathrm{p}=0.039$ ). Simple and multiple logistic regression analysis revealed none of the variables predicted survival in WF cats.

\section{Discussion}

Our findings indicate that naturally occurring WF-related thermal burn injuries and smoke exposure resulted in the priming of platelets that potentiated platelet activation and shedding of PDMV in cats. Persistent platelet activation in response to AA was also independently associated with intracardiac thrombus in this population of cats. 
The findings of this study open a dialogue about the impact of natural wildfires and air pollution on the well-being of humans, wildlife, and domestic animals. There is mounting epidemiologic data relating poor air quality from wildfires to growing incidences of human cardiovascular diseases, CHF, out-of-hospital cardiac arrest and cerebrovascular events ${ }^{2,3,5,21-26}$. While robust data exists in both animal models and human studies documenting the procoagulant effects of fine particulate matter $\left(\mathrm{PM}_{2.5}\right)$ exposure, the role of platelets in propagating a hypercoagulable state and subsequent thrombosis in a natural setting has never been established. Our data show that wildfire-related injuries and smoke inhalation results in the priming of platelets. Priming occurs when an initial stimulus, known as a primer, counteracts the intrinsic regulatory mechanisms to potentiate the activation of platelets upon subsequent exposure to physiologic agonists. This causes unrestrained platelet activation, aggregation, and sustained thrombus formation ${ }^{27}$. Similar findings have also been shown in experimental models of $\mathrm{PM}_{2.5}$ exposure. Studies demonstrated that mice exposed to concentrated ambient PM not only have elevated inflammatory cytokines and PMV, but also have platelets with increased fibrinogen binding and sensitivity to thrombin, both of which are indicative of platelet priming ${ }^{17,28}$. While it is difficult to ascertain the extent of $\mathrm{PM}_{2.5}$ inhalation in our population of cats, forecast models in Northern California's Butte County and the surrounding regions during the time of enrollment estimated toxic levels of $\mathrm{PM}_{2.5} \cdot{ }^{29}$ In addition, cats rescued from the California Camp Fire originated from an urbanized environment and likely inhaled smoke comprised of noxious gases and potent $\mathrm{PM}_{2.5}$.

Although our study did not assess the presence of platelet primers in cats with WF injuries, increased circulating primers such as thrombopoietin, epinephrine, and vascular endothelial growth factors (VEGFs) have been well documented in human burned patients ${ }^{30-32}$. Other less well described primers such as dioxins, benzimidazoles and lipopolysaccharide (LPS) are known $\mathrm{PM}_{2.5}$-bound trace elements. Dioxins has previously been shown to prime human platelets via the aryl hydrocarbon receptor by activating the phospholipase $\mathrm{C}$ and p38 MAPK pathway ${ }^{33}$. PM 2.5 -bound LPS can also modulate splenocyte immune response and exacerbate airway inflammation by TLR-4 and -2 pathways ${ }^{34-36}$. We previously showed that ex vivo exposure of canine platelets to LPS primes platelets to respond to ADP via platelet TLR-4 to potentiate the cyclooxygenase pathway leading to excessive release of thromboxane $A_{2}(T x A 2)^{37}$. Similarly, TLR-4 mediated priming in human platelets also require excess production of eicosanoids. TLR4 is also a receptor for danger associated molecular patterns like high mobility group box-1 and extracellular histones that are released in response to systemic inflammation and tissue injuries. Platelet TLR4 may play a role in platelet priming in wildfire-related injuries but further studies are needed.

While exogenous addition of AA generally resulted in platelet inhibition in our study, persistent platelet response to AA was found to be independently associated with intracardiac thrombosis. Two human studies of flame-related or electrical burn events found a similar response to AA stimulation; the mechanism for this response, however, has not been elucidated ${ }^{7,38}$. AA is a phospholipid released by the soluble enzyme phospholipase $A 2$ and is then catalyzed by cyclooxygenase (COX) to produce $\mathrm{TxA}_{2}$, prostacyclin $\left(\mathrm{PGI}_{2}\right)$ and prostaglandin $\mathrm{E} 2\left(\mathrm{PGE}_{2}\right)$. While $\mathrm{TxA}_{2}$ enhances platelet activation via TPa 
receptors, $\mathrm{PGI}_{2}$ and $\mathrm{PGE}_{2}$ inhibit platelets by binding to their respective EP2 and EP4 receptors, leading to increased intracellular cyclic adenosine monophosphate levels ${ }^{14,39}$. There are two plausible explanations to this observed biphasic response to AA. First, prolonged exposure of high-dose AA in our study likely inhibited platelets by inducing high concentrations of in $\mathrm{PGE}_{1}$ and $\mathrm{PGE}_{2}$ in the platelet milieu ${ }^{40}$. Second, it is conceivable that circulating platelets may encounter platelet primers released or shed from the endothelium or established thrombi as they pass through the LA. Platelet-derived primers released from activated platelets may also further potentiate platelet response. For example, low concentrations of $\mathrm{PGE}_{2}$ released from activated platelets have been shown to potentiate platelet aggregation by priming phosphokinase $C$ and inhibiting adenylcyclase ${ }^{41,42}$. This may shift the inhibitory pathway of $A A$ to a stimulatory one that favors $\mathrm{TxA}_{2}$ production. Due to the inhibitory effect noted, we did not evaluate platelet response to high-dose AA in cats in the $\mathrm{CC}$ and $\mathrm{HCM}$ groups. We, therefore, cannot conclude if platelet activation in response to high-dose $A A$ is a unique finding in those with WF injuries. AA was chosen as an agonist to evaluate platelet priming in WF group to determine if aspirin, a COX-1 inhibitor, would be indicated in those with intracardiac thrombosis. Given the biphasic response to AA noted in our study, it appears that aspirin may only be appropriate in a subset of cats with documented thrombosis, while aspirin as a thromboprophylaxis could promote thrombosis by inhibiting prostaglandin synthesis. Further studies are needed to investigate the efficacy of anti-platelet therapies in preventing thrombosis in cats with WF-related injuries.

In agreement with previous studies, we found that cats with subclinical HCM had evidence of increased platelet priming compared to healthy controls. We chose cats with subclinical HCM as a control group since previous studies had found that subclinical HCM, LV cavity obliteration and decreased diastolic function were associated with increased platelet priming, platelet activation and PDMV shedding ${ }^{18-20,43}$. In addition, compromised LA function secondary to HCM, which leads to blood flow stasis, has also been proposed to facilitate the formation of SEC $\pm T$ and cardiogenic thromboembolism ${ }^{44}$. Interestingly, the presence of MT was not associated with platelet activation while SEC $\pm T$ also occurred in the absence of LA dysfunction among WF cats. In addition, cats in WF group had platelets with more exaggerated response to agonists compared to those in $\mathrm{HCM}$ group. These findings suggest that increased platelet priming and SEC $\pm T$ in WF were unlikely to be related to their transient cardiomyopathies. The myocardial changes noted in WF cats could be due to myocardial edema, myocarditis from systemic inflammation or catecholamine surges following WF injuries. ${ }^{1}$ Increased platelet priming and activation could, therefore, occur in parallel with the observed transient cardiopathies as a result of systemic inflammation due to smoke inhalation and thermal burn injuries. In contrast, $\mathrm{HCM}$, which causes primary myocardial structural changes, augments the components of Virchow's triad. While cats in the WF group may have similar echocardiographic indications like LA dilation or presence of SEC $\pm T$ to initiate anti-platelet therapy, it is important to note the differences in the underlying causes of thrombosis between HCM and wildfire injuries ${ }^{45,46}$. For that reason, aspirin, which was shown to be inferior to clopidogrel in preventing recurrent thrombosis in HCM cats, may be a more appropriate antiplatelet therapy than clopidogrel in cats with wildfire-related injuries. 
While our study did not evaluate specific inflammatory biomarkers, increased neutrophil counts found in the WF group is indicative of a proinflammatory state. Increases in interleukin-10 and macrophage colony-stimulating factor, alongside increases in circulating PDMV have been in found in mice exposed to PM and human burn victims ${ }^{17,38}$. Similarly, cats in the WF group had substantial elevation in circulating PDMV, which may further promote thrombosis by facilitating the assembly of thrombin generating complexes on externalized PS ${ }^{14,15,47}$. P-selectin, found on the membrane of platelet a-granules, is expressed on the plasma membrane upon platelet activation and degranulation. In mice, upregulation of platelet P-selectin promotes platelet-leukocyte interaction by binding to P-Selectin Ligand-1 on leukocytes, thus triggering the upregulation of tissue factor on leukocytes and initiating the extrinsic coagulation cascade in the absence of vascular injury ${ }^{15,48,49}$. In one study that performed intratracheal instillation of carbon nanotubes as a surrogate for air pollution, P-selectin neutralization abrogated platelet-leukocyte aggregations and procoagulant microvascular tissue factor activity in mice ${ }^{50}$. This highlights the role that platelet P-selectin may play in linking hemostasis and inflammation following wildfire injuries. However, the molecular mechanisms involved in platelet-leukocyte interaction are highly species dependent and further investigations are needed to further elucidate the role of platelet P-selectin in thrombosis and inflammation in cats.

This study has several limitations. First, we did not assess secondary and tertiary hemostasis so the impact of wildfire injuries on global coagulation cannot be confirmed. Second, pro-inflammatory mediators were not measured during this study. Also, due to the emergency presentation of patients, the duration and extent of injuries and smoke inhalation in these cats could not be accurately determined. However, attempts were made to standardize the time of sample collection following presentation. In addition, the lack of a validated system to estimate the extent of burn severity in cats might have greatly limited our ability to identify associations between thrombosis and burn severity. Our assessment was largely based on review of medical records and subjective assessment of photographic documentation hence total body surface area affected could not be estimated in our cohort of animals. In human patients, estimated burn injuries affecting greater than $6 \%$ of total body surface area were shown to be associated with systemic hypercoagulability and early coagulopathy ${ }^{51}$. Furthermore, the small number of cats in the WF group could have led to type II error causing the lack of detectable associations between myocardial changes and SEC $\pm \mathrm{T}$.

In conclusion, marked platelet activation and shedding of PDMV due to platelet priming is present following naturally occurring wildfire exposure and thermal burn injuries in a population of domestic cats. This may exacerbate systemic inflammation and hemostatic derangement resulting in cardiovascular events and thrombosis, which is associated with persistent platelet response to AA. Aspirin may be the preferred anti-platelet therapy of choice for a subset of feline patients with confirmed thrombosis but further studies are required to delineate additional mechanisms between inflammation and thrombosis, especially in regard to the COX-1 pathway.

\section{Materials And Methods}




\section{Animals and Study Design}

The study was a prospective observational case-control study. Clinical cats were enrolled in accordance with the Institutional Animal Care and Use Committee (IACUC) at the University of California, Davis. Those exposed to wildfires were treated according to the best veterinary standard of care and those that served as controls were enrolled as part of IACUC protocols \#20095 and \#21037. All experimental protocols were approved by the IACUC of the University of California, Davis and all methods were carried out in compliance with the ARRIVE guidelines (http://www.nc3rs.org.uk/page.asp?id=1357) and regulations for care and use of laboratory animals. Informed owner consent was obtained prior to enrolling all client-owned cats in this study.

\section{Clinical cats with thermal and smoke inhalation injury}

Twenty-nine cats presented to the Veterinary Medical Teaching Hospital of the University of California, Davis for treatment of thermal burn injury and smoke inhalation due to the 2018 California Camp Fire and were enrolled in this arm of the study. The severity of thermal burn injuries was retrospectively scored as 1 (mild), 2 (moderate) or 3 (severe) based on clinical records and photographic documentation as previously described ${ }^{1}$. Medical treatment of wildfire-exposed cats was left to the clinicians' discretion and detailed as previously reported ${ }^{1}$. All samples were collected within 7 days after presentation.

\section{Healthy cats with or without subclinical hypertrophic cardiomyopathy}

Thirty-two student, staff or client-owned cats were recruited during the period of October 2018 to June 2019. All cats received a physical examination, complete blood count (Abaxis, Union City, CA), total thyroxine (Abaxis, Union City, CA) and blood pressure measurement via Doppler with sphygmomanometery prior to echocardiography. Cats were excluded if they had a previous history of $\mathrm{CHF}$, uncooperative temperament, apparent systemic diseases including abnormal findings in physical examination, hematology, systemic hypertension (systolic blood pressure $>160 \mathrm{mmHg}$ ) or hyperthyroidism $(>4.8 \mu \mathrm{g} / \mathrm{dl})$. All cats were screened and confirmed for the presence of HCM with echocardiography and assigned to the HCM or CC groups. Oral administration of gabapentin (50 to 100 $\mathrm{mg}$ ) prior to enrollment was permitted.

\section{Echocardiography}

Transthoracic echocardiographic examinations were performed by board-certified veterinary cardiologists (JAS, CGH) or cardiology trainees (ANS, YU) under direct supervision of a board-certified cardiologist. Standard imaging planes were used with the patient in right and left lateral recumbency. One of two machines were used with a 12-4mHz sector array transducer (Philips iE33 Ultrasound, Philips Health Care, 
Andover, MA). All measurements were evaluated by the investigators using commercially available software (Syngo Dynamic Workspace 10.0.01, Siemens Medical Solutions, Malvern, PA).

Diagnosis of HCM was made based on identification of idiopathic left ventricular hypertrophy characterized by regional or global end-diastolic wall thickening $\geq 6 \mathrm{~mm}$, determined by 2-dimenional or $m$-mode echocardiography while avoiding inclusion of moderator band insertion sites ${ }^{52}$. Myocardial thickening was defined as a diagnostic wall thickness $\geq 6 \mathrm{~mm}$. LA enlargement was measured in the right parasternal short axis basilar view and defined as an LA/Ao of $\geq 1.6$. The LAuV was measured in the oblique left apical parasternal long axis view with pulsed wave Doppler sample volume at the entrance to the left auricle ${ }^{44}$. A LAuV of $>47 \mathrm{~cm} / \mathrm{s}$ was considered normal ${ }^{44}$. M-mode was used to acquire measurements of LV internal dimension at end-diastole (LVIDd) and LV internal dimension at end-systole (LVIDs) and fractional shortening FS\%) was calculated using the equation:

\section{FS $(\%)=\frac{(\text { LVIDd }- \text { LVIDs })}{\text { LVIDd }} \times 100$}

CHF in the WF group was diagnosed by a combination of clinical signs, echocardiographic findings of LA enlargement with the presence of pericardial and/or pleural effusion and radiographic findings consistent of cardiogenic pulmonary edema.

\section{Blood sample collection}

Blood was collected from the jugular or medial saphenous vein with a $22 \mathrm{G}$ or $23 \mathrm{G}$ butterfly catheter and divided among lithium-heparin tubes and 3.2\% trisodium citrate tubes immediately after collection. Citrated blood samples were processed within 60 to 90 minutes after collection.

\section{Generation of platelet rich plasma}

Citrated blood was transferred to polypropylene tubes and rested for 30 minutes at $37^{\circ} \mathrm{C}$ to facilitate red cell sedimentation. The samples were then centrifuged at room temperature at $200 \mathrm{xg}$ for 5 mins, no brakes). Platelet rich plasma (PRP) was then separated and rested for an additional 30 minutes at $37^{\circ} \mathrm{C}$ prior to analysis. Swirling characteristic of the PRP was noted to ensure that platelets maintained their discoid shape. A platelet count of the PRP was attained with the automated blood cell analyzer (HM5, Abaxis, Union City, CA) and confirmed by blood smear evaluation when indicated.

\section{Detection of platelet P-selectin by flow cytometry}


PRP was diluted with Tyrodes HEPES buffer ( $\mathrm{pH} 7.2,5 \mathrm{mM}$ dextrose, without divalent cations) to a final concentration of $1 \times 10^{7}$ platelets $/ \mathrm{ml}$. Platelets were either unstimulated (resting) or activated in the

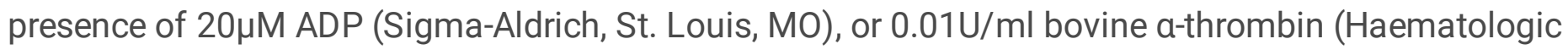
Technologies, Essex Junction, VT) for 15 minutes at $37^{\circ} \mathrm{C}$. Platelet P-selectin was detected by fluorescein isothiocyanate-conjugated rat anti-mouse monoclonal antibody to $\operatorname{CD} 62 \mathrm{P}(1: 200$, clone:RB40.34, BD Pharmingen, San Jose, $C A)$. Platelet integrin- $\beta 3$ was identified using allophycocyanin-conjugated mouse anti-human monoclonal antibodies to CD61 (1:500, Clone: VI-PL2, eBioscience, San Diego, CA). Samples were subsequently incubated in the dark at $37^{\circ} \mathrm{C}$ degrees for 45 minutes before fixation with $1 \%$ paraformaldehyde at room temperature for 30 minutes. Samples were then analyzed using a 5-color flow cytometer (FC500, Beckmann Coulter, Miami, FL).

Platelets were identified by CD61-positive events as well as forward- and side-scatter characteristics using $0.9 \mu \mathrm{m}$ and $3.0 \mu \mathrm{m}$ calibration beats as previously described ${ }^{37}$. Gating was established using fluorescence minus one controls consisting of unstimulated or activated platelets labelled with either 1 of the antibodies (Fig. 1A,B). Fluorescence compensation was applied using anti-mouse isotype controls conjugated to matched fluorophores (BD Biosciences, San Diego, $C A$ ) in identical experimental conditions for calculations of compensation matrixes. Flow cytometry data were analyzed using commercially available software (Flowjo, TreeStar Inc, Ashland, OR). Surface P-selectin expression on platelets was quantified as percentage of CD62P-positive events out of 10,000 platelets or median fluorescence intensity (MFI), an indicator of protein density.

In WF cats, platelet activation in response to $1 \mu \mathrm{M}$ AA (Roche) was assessed by treating PRP with $1 \mu \mathrm{M} \mathrm{AA}$ for 15 minutes at $37^{\circ} \mathrm{C}$. Unexpectedly, AA led to platelet inhibition as indicated by a significant decrease in P-selectin positive platelets compared to resting platelets (3.87\%; IQR: $0.41-32.40$ vs. $63.80 \%$ : IQR: $28.75-71.35 ; p<0.0001)$.

\section{Detection of platelet-derived microvesicles by flow cytometry}

Platelet poor plasma (PPP) from $28 \mathrm{WF}$ and 8 cats from the CC group was generated from PRP by further centrifugation at $5000 \mathrm{x} \mathrm{g}$ for 15 minutes. Supernatant was flash frozen in liquid nitrogen and stored at $-80^{\circ} \mathrm{C}$ until further analysis. PPP was thawed at room temperature and only the top $90 \%$ of PPP was used for analysis. Positive controls were generated by treating PRP $\left(1 \times 10^{7} / \mathrm{ml}\right)$ from healthy controls with $2 \mathrm{mM}$ calcium chloride $\left(\mathrm{CaCl}_{2}\right)$ over a 60 -minute interval $\left(1 \mathrm{mM} \mathrm{CaCl}_{2}\right.$ every 30 minutes $)$ and $2.5 \mu \mathrm{M}$ A23187 (Millipore Sigma, Burlington, MA) for 15 minutes at $37^{\circ} \mathrm{C}$ (Fig. 5B,C). To detect PMV, PPP was incubated in the dark at room temperature for 30 minutes with phycoerythrin-conjugated monoclonal mouse IgG1 antibodies to CD61 (1:10, Clone: VI-PL2, eBioscience, San Diego, CA) and fluorescein isothiocyanate-conjugated Annexin-V (1:32), diluted in binding buffer ( $\mathrm{pH} 7.4,100 \mathrm{mM}$ HEPES, $140 \mathrm{mM}$ $\mathrm{NaCl}, 25 \mathrm{mM} \mathrm{CaCl}_{2}$ (BD Biosciences, San Jose, CA). Prior to analysis by flow cytometry (FC-500, Beckman Coulter, Brea, CA), samples were further diluted in binding buffer (1:10) and agitated on a vortex for 5 
seconds. Gating to quantify PMV were set by CD61-positive events and Annexin-V positive scatter characteristics using $0.5 \mu \mathrm{m}$ and $3.0 \mu \mathrm{m}$ calibration beads (Megamix, Biocytex) as previously described (Fig. 4) ${ }^{20}$. PMV was quantified as a percentage of CD61- and Annexin-V positive events and also by absolute counts per $\mu$ l of PPP. Flow cytometry data were analyzed by commercially available software (Beckman Coulter, Brea, CA).

\section{Statistics}

Assuming biologic variability of $20 \%$, calculations from our preliminary data and standard deviation of data gathered from the 29 WF cats showed that a sample size of at least 8 normal cats and $20 \mathrm{HCM}$ cats would be required to demonstrate significant changes with an alpha priori of 0.05 and a power of $80 \%$. Data was assessed for normality with Shapiro-Wilk normality test. Categorical data between groups were compared using a Fisher's exact test or Chi-square analysis. Continuous data between groups were analyzed using either one-way ANOVA or Kruskal-Wallis test, followed by Dunnett's or Dunn's multiple comparisons test as appropriate. Unpaired continuous data were analyzed using student t-test for normally distributed data or Mann-Whitney test for non-parametric data. Spearman's rank correlation analysis was performed between resting and activated platelets. Univariate and multiple logistic regression analyses were performed to predict the development of clot formation and survival by burn severity (1,2 or 3), platelet count, neutrophil count and degree of platelet activation (P-selectin MFI) and the magnitude of response to ADP, AA and thrombin based on MFI fold change $\left(\log _{10} \mathrm{MFI}\right.$ activated $-\log _{10}$ $\mathrm{MFI}_{\text {rest }}$ ) on a $\log _{10}$ scale. A forward selection technique was performed by including the variable with $\mathrm{P}$ values $<0.1$. To test the adequacy of the multivariable regression model, a Hosmer-Lemeshow goodness of fit test was performed. Normally distributed data were presented as mean \pm standard deviation while non-parametric data were presented as median and interquartile range (IQR). Data were analyzed using commercially available software (Prism 8, Graphpad, San Diego, CA and STATA v15.1, College Station, Texas).

\section{References}

1. Sharpe, A. N., Gunther-Harrington, C. T., Epstein, S. E., Li, R. H. \& Stern, J. A. Cats with thermal burn injuries from California wildfires show echocardiographic evidence of myocardial thickening and intracardiac thrombi. Sci. Rep, 10, 2648 (2020).

2. Wettstein, Z. S. et al. Cardiovascular and Cerebrovascular Emergency Department Visits Associated With Wildfire Smoke Exposure in California in 2015.J Am Heart Assoc7, (2018).

3. Cascio, W. E. Wildland fire smoke and human health. Science of The Total Environment, 624, 586595 (2018).

4. Tinling, M. A., West, J. J., Cascio, W. E., Kilaru, V. \& Rappold, A. G. Repeating cardiopulmonary health effects in rural North Carolina population during a second large peat wildfire. Environ. Health, 15, 12 (2016). 
5. Haikerwal, A. et al. Impact of Fine Particulate Matter (PM2.5) Exposure During Wildfires on Cardiovascular Health Outcomes. Journal of the American Heart Association4, (2015).

6. Ball, R. L. et al. Burn-Induced Coagulopathies. SHOCK, 54, 154-167 (2020).

7. Wade, C. E. et al. Upon admission coagulation and platelet function in patients with thermal and electrical injuries. Burns, 42, 1704-1711 (2016).

8. Wiegele, M. et al. Thrombin generation in patients with severe thermal injury. Burns, 45, 54-62 (2018).

9. Marck, R. E. et al. Activation, function and content of platelets in burn patients., 30, 1-7 (2018).

10. Chai, J. et al. A Novel Model of Burn-Blast Combined Injury and Its Phasic Changes of Blood Coagulation in Rats. Shock, 40, 297-302 (2013).

11. Midura, E. F. et al. Impact of Platelets and Platelet-Derived Microparticles on Hypercoagulability Following Burn Injury. SHOCK, 45, 82-87 (2016).

12. Deppermann, C. \& Kubes, P. Start a fire, kill the bug: The role of platelets in inflammation and infection. Innate Immunity, 24, 335-348 (2018).

13. Koupenova, M., Clancy, L., Corkrey, H. A. \& Freedman, J. E. Circulating Platelets as Mediators of Immunity, Inflammation, and Thrombosis. Circulation research, 122, 337-351 (2018).

14. Jurk, K., Kehrel, B. E. \& Platelets Physiology and Biochemistry. Semin Thromb Hemost, 31, 381-392 (2005).

15. Mezger, M. et al. Platelets and Immune Responses During Thromboinflammation. Front Immunol, 10, 1731 (2019).

16. Lopez, E., Srivastava, A. K., Pati, S., Holcomb, J. B. \& Wade, C. E. Platelet-Derived Microvesicles. SHOCK, 49, 243-248 (2018).

17. Wilson, D. W. et al. Exposure of mice to concentrated ambient particulate matter results in platelet and systemic cytokine activation. Inhalation Toxicol, 22, 267-276 (2010).

18. Holme, P. A. et al. Shear-Induced Platelet Activation and Platelet Microparticle Formation at Blood Flow Conditions as in Arteries With a Severe Stenosis. Arteriosclerosis Thrombosis Vasc Biology, 17, 646-653 (1997).

19. Tablin, F. et al. Platelet Activation in Cats with Hypertrophic Cardiomyopathy. Journal of Veterinary Internal Medicine, 28, 411-418 (2014).

20. Li, R. H. L., Stern, J. A., Ho, V., Tablin, F. \& Harris, S. P. Platelet Activation and Clopidogrel Effects on ADP-Induced Platelet Activation in Cats with or without the A31P Mutation in MYBPC3. Journal of Veterinary Internal Medicine, 30, 1619-1629 (2016).

21. Adetona, O. et al. Review of the health effects of wildland fire smoke on wildland firefighters and the public. Inhal Toxicol, 28, 95-139 (2016).

22. Reid, C. E. et al. Critical Review of Health Impacts of Wildfire Smoke Exposure. Environ. Health Perspect, 124, 1334-1343 (2016). 
23. DeFlorio-Barker, S., Crooks, J., Reyes, J. \& Rappold, A. G. Cardiopulmonary Effects of Fine Particulate Matter Exposure among Older Adults, during Wildfire and Non-Wildfire Periods, in the United States 2008-2010. Environ. Health Perspect, 127, 037006 (2019).

24. Dennekamp, M. et al. Forest Fire Smoke Exposures and Out-of-Hospital Cardiac Arrests in Melbourne, Australia: A Case-Crossover Study. Environ Health Persp, 123, 959-964 (2015).

25. Rappold, A. G. et al. Peat Bog Wildfire Smoke Exposure in Rural North Carolina Is Associated with Cardiopulmonary Emergency Department Visits Assessed through Syndromic Surveillance. Environ Health Persp, 119, 1415-1420 (2011).

26. Delfino, R. J. et al. The relationship of respiratory and cardiovascular hospital admissions to the southern California wildfires of 2003. Occup Environ Med, 66, 189 (2009).

27. Gresele, P., Falcinelli, E. \& Momi, S. Potentiation and priming of platelet activation: a potential target for antiplatelet therapy. Trends Pharmacol Sci, 29, 352-360 (2008).

28. Emmerechts, J. et al. Thrombogenic changes in young and old mice upon subchronic exposure to air pollution in an urban roadside tunnel. Thrombosis and Haemostasis, 108, 756-768 (2012).

29. Butte county air quality management district. Air Quality Summary for 2018 and 2018-2019 Check Before You Light Program Summary. http://bcaqmd.org/wp-content/uploads/Final-2018-Annual-AirQuality-Report-and-2018-2019-CBYL-Review.pdf

30. Lupia, E. et al. Elevated thrombopoietin in plasma of burned patients without and with sepsis enhances platelet activation. J Thromb Haemost, 7, 1000-1008 (2009).

31. Infanger, M. et al. Vascular endothelial growth factor serum level is strongly enhanced after burn injury and correlated with local and general tissue edema. Burns, 30, 305-311 (2004).

32. Williams, F. N. et al. Changes in Cardiac Physiology After Severe Burn Injury. J Burn Care Res, 32, 269-274 (2011).

33. Pombo, M., Lamé, M. W., Walker, N. J., Huynh, D. H. \& Tablin, F. TCDD and omeprazole prime platelets through the aryl hydrocarbon receptor (AhR) non-genomic pathway. Toxicol. Lett, 235, 28-36 (2015).

34. He, C. et al. Lipopolysaccharide levels adherent to PM2.5 play an important role in particulate matter induced-immunosuppressive effects in mouse splenocytes. J Appl Toxicol, 38, 471-479 (2018).

35. He, M. et al. Urban PM2.5 exacerbates allergic inflammation in the murine lung via a TLR2/TLR4/MyD88-signaling pathway. Sci Rep-uk, 7, 11027 (2017).

36. Fu, H. et al. PM2.5 Exposure Induces Inflammatory Response in Macrophages via the TLR4/COX2/NF-KB Pathway. Inflammation, 43, 1948-1958 (2020).

37. Li, R. H., Nguyen, N. \& Tablin, F. Canine platelets express functional Toll-like receptor-4: lipopolysaccharide-triggered platelet activation is dependent on adenosine diphosphate and thromboxane A2 in dogs. BMC Veterinary Research, 15, 245 (2019).

38. Schmidt, A. et al. The role of inflammation, platelets, and microparticles/nanoparticles in burn wounds. American Burn Association 51st Annual Meeting. Abstract 348(2019) 
39. Nagy, Z. \& Senis, Y. A.. Platelet inhibitory receptors in Platelets (ed. Michelson, A).279-293(Academic Press, 2019)

40. Rukoyatkina, N. et al. Multifaceted effects of arachidonic acid and interaction with cyclic nucleotides in human platelets. Thromb Res, 171, 22-30 (2018).

41. Vezza, R., Roberti, R., Nenci, G. \& Gresele, P. Prostaglandin E2 potentiates platelet aggregation by priming protein kinase C., 82, 2704-2713 (1993).

42. Fabre, J. E. et al. Activation of the murine EP3 receptor for PGE2 inhibits CAMP production and promotes platelet aggregation. J Clin Invest, 107, 603-610 (2001).

43. Bédard, C., Lanevschi-Pietersma, A. \& Dunn, M. Evaluation of coagulation markers in the plasma of healthy cats and cats with asymptomatic hypertrophic cardiomyopathy. Vet Clin Path, 36, 167-172 (2007).

44. Schober, K. E. \& Maerz, I. Doppler echocardiographic assessment of left atrial appendage flow velocities in normal cats. Journal of Veterinary Cardiology, 7, 15-25 (2005).

45. Hogan, D. F. et al. Secondary prevention of cardiogenic arterial thromboembolism in the cat: The double-blind, randomized, positive-controlled feline arterial thromboembolism; clopidogrel vs. aspirin trial (FAT CAT). Journal of veterinary cardiology: the official journal of the European Society of Veterinary Cardiology, 17 (Suppl 1), S306-17 (2015).

46. Fuentes, V. L. et al. ACVIM consensus statement guidelines for the classification, diagnosis, and management of cardiomyopathies in cats. J Vet Intern Med, 34, 1062-1077 (2020).

47. Melki, I., Tessandier, N., Zufferey, A. \& Boilard, E. Platelet microvesicles in health and disease., 28, 1-8 (2017).

48. Ivanov, I. I., Apta, B. H. R., Bonna, A. M. \& Harper, M. T. Platelet P-selectin triggers rapid surface exposure of tissue factor in monocytes. Sci Rep-uk, 9, 13397 (2019).

49. Thomas, M. \& Storey, R. The role of platelets in inflammation. Thromb Haemostasis, 114, 449-458 (2015).

50. Nemmar, A. et al. Enhanced peripheral thrombogenicity after lung inflammation is mediated by platelet-leukocyte activation: role of P-selectin. J Thromb Haemost, 5, 1217-1226 (2007).

51. Haren, R. M. V. et al. Hypercoagulability after burn injury. Journal of Trauma and Acute Care Surgery, 75, 37-43 (2013).

52. Payne, J. R., Brodbelt, D. C. \& Fuentes, V. L. Cardiomyopathy prevalence in 780 apparently healthy cats in rehoming centres (the CatScan study). Journal of Veterinary Cardiology, 17, S244-S257 (2015).

\section{Figures}




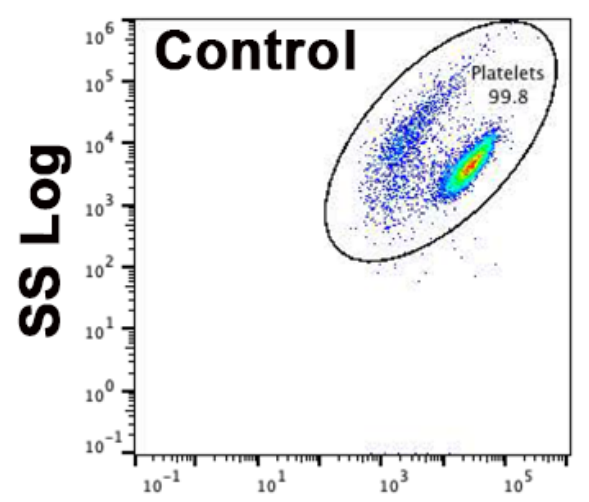

FS Log

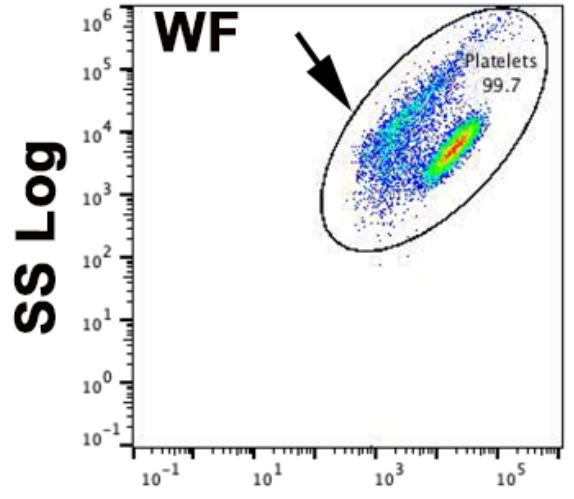

FS Log

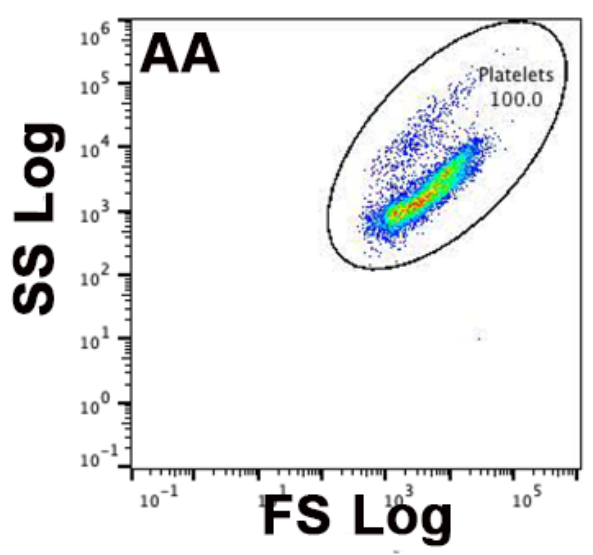

ADP

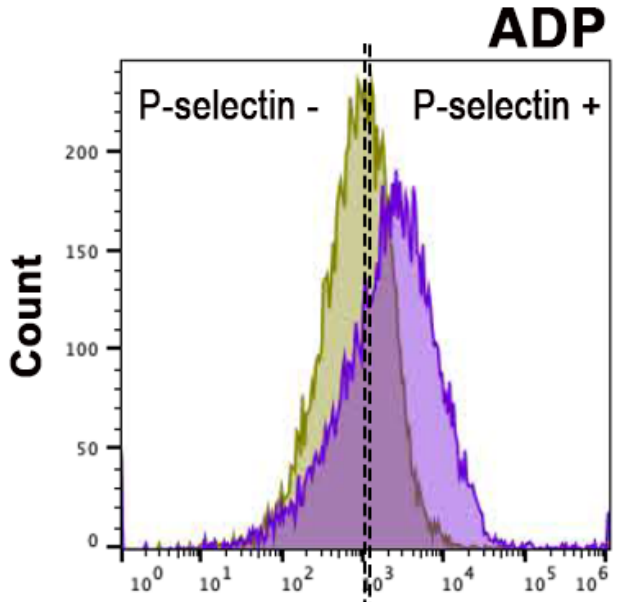

FITC' Log

=E WF Log Control

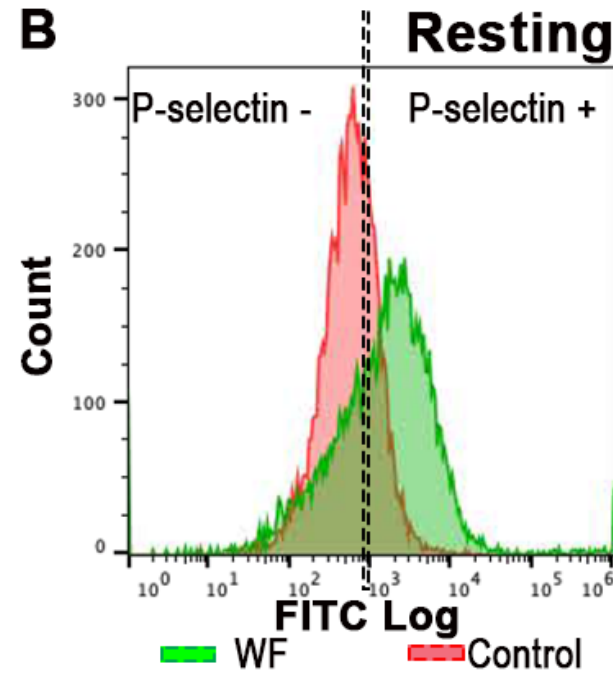

as WF Control

\section{Figure 1}

Representative scatter plot diagrams and histograms of flow cytometric analysis of platelets in 1 cat with wildfire (WF) related injuries and 1 healthy control cat. $(A, B)$ Platelets were identified by forward (FS) and side scatter (SS) properties. Note the shift in scatter properties (arrows) in platelets from a cat in WF group compared to those in the healthy control indicating shape change and degranulation. Arachidonic acid (AA)-treated platelets in a WF group cat resulted in a shift in scatter properties indicative of intact granules and size. (B) Representative histograms illustrating the number of platelets expressing $P$ selectin in the absence (resting) or presence of ADP in a WF cat and healthy control. Note the upregulation in P-selectin in either resting or ADP-activated platelets in the WF cat compared to healthy control. 

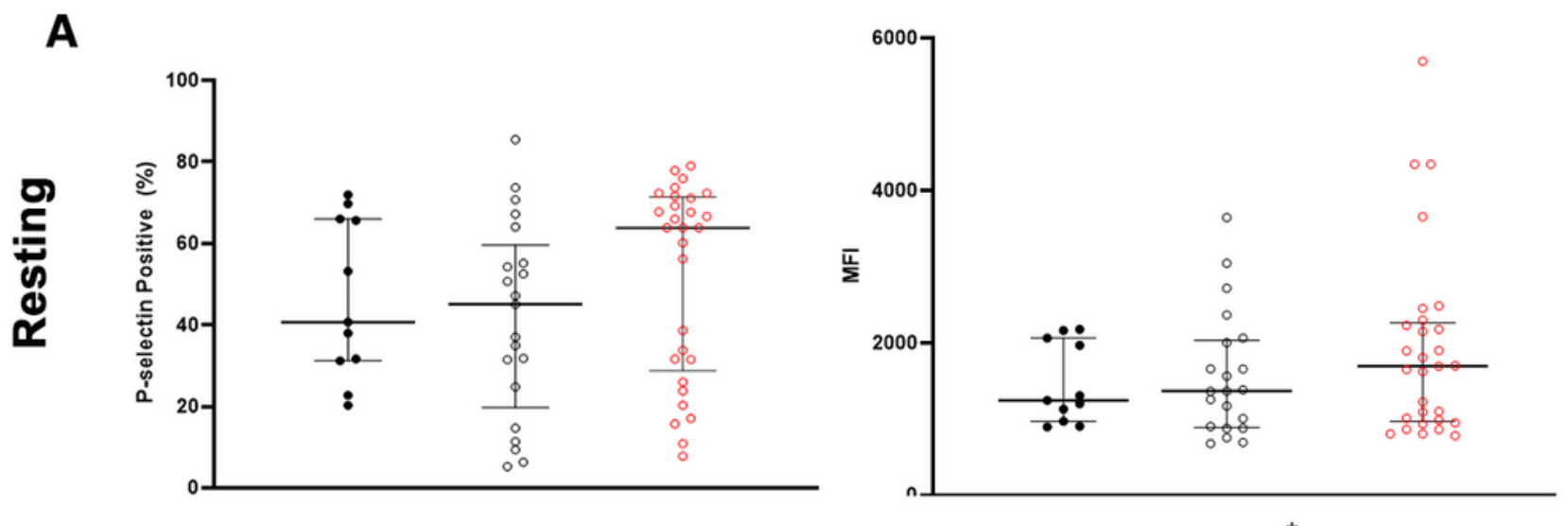

B
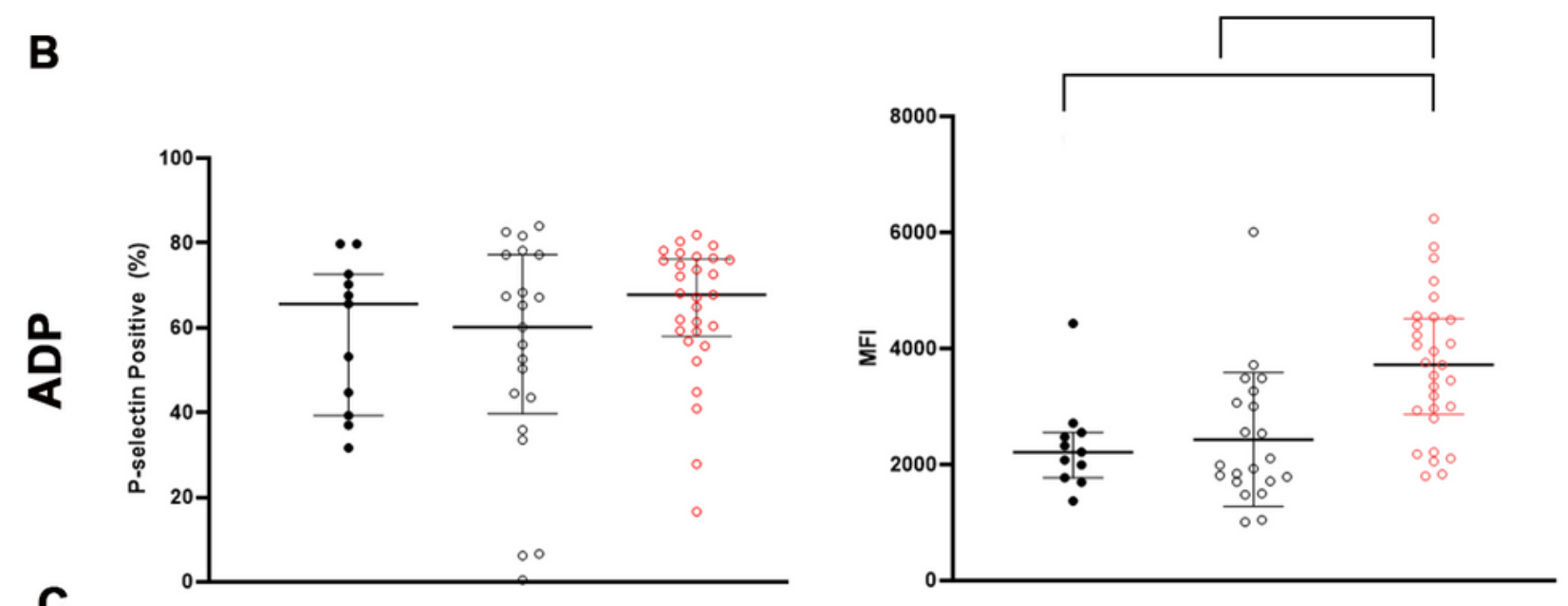

C
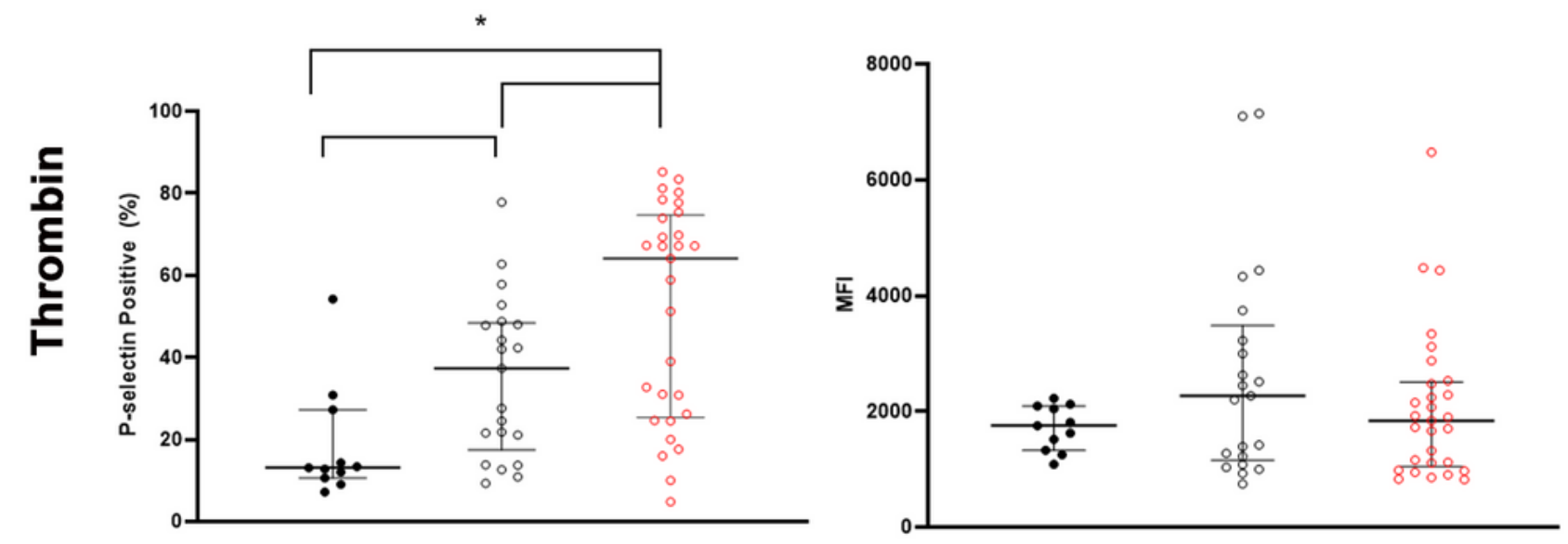

сс $\bigcirc$ нсм $\bigcirc \mathbf{W F}$

\section{Figure 2}

Increased platelet priming in cats with naturally occurring wildfire injuries and smoke exposure. Platelet activation, measured as a percentage of P-selectin positive or P-selectin median florescence intensity (MFI), was evaluated by flow cytometry in 29 cats exposed to wildfires (WF), 21 cats with subclinical hypertrophic cardiomyopathy (HCM) and 11 control cats without $\mathrm{HCM}(\mathrm{CC})$. Platelet rich plasma was measured at resting (unstimulated) (A) or in the presence of ADP (B) or thrombin (C). (A) No difference in 
platelet activation at rest was found among the 3 groups. $(B, C)$ WF cats had increased platelet activation in response to ADP (MFI) and thrombin (\% positive) compared to cats in the HCM and CC groups. (C) Cats with HCM had increased response to thrombin compared with cats in the CC group, HCM: cats with subclinical HCM and WF: cats with wildfire injuries. * denotes $\mathrm{P}<0.002$

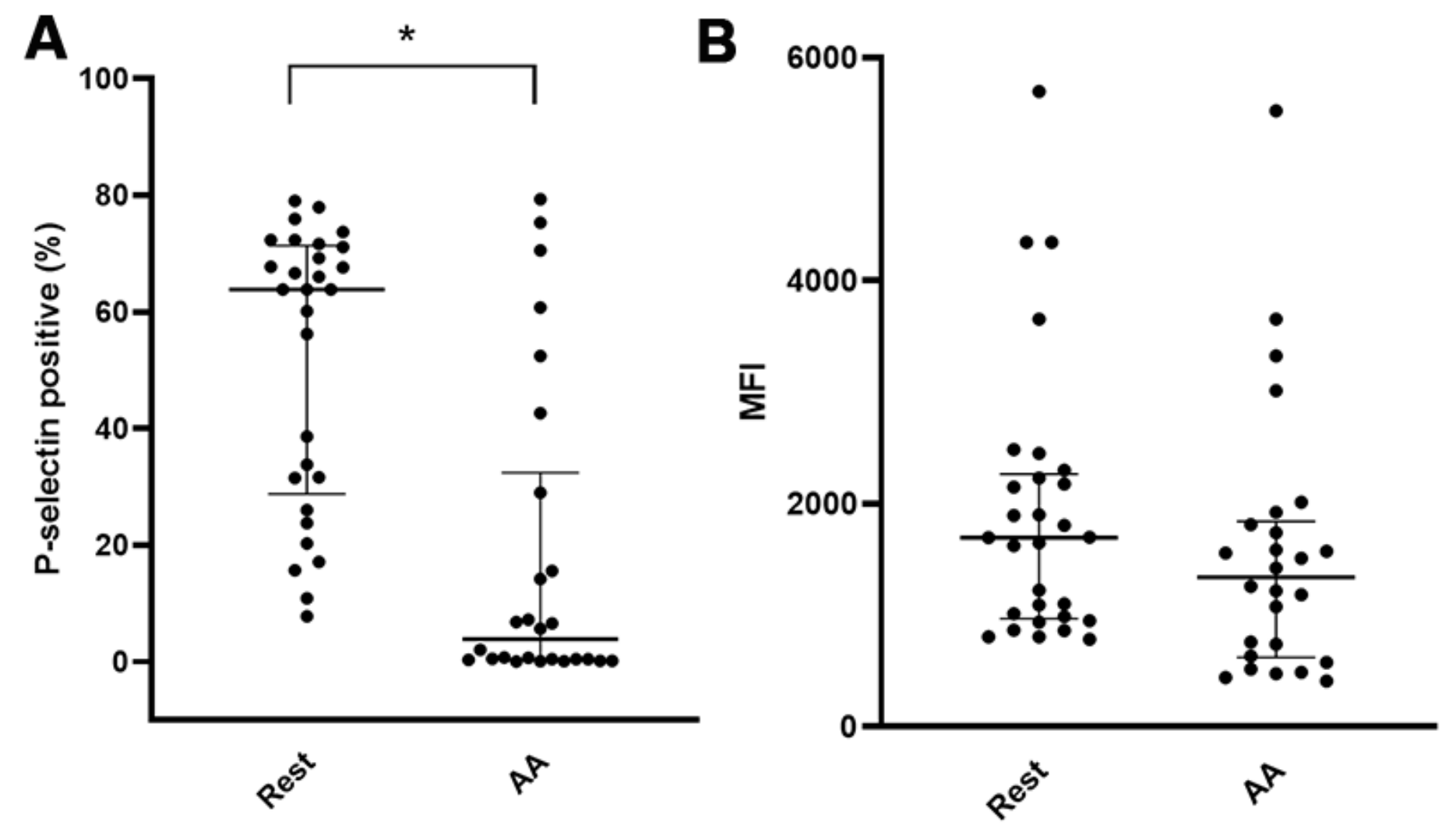

Figure 3

Arachidonic acid results in platelet inhibition. Platelet activation was assessed in 29 cats with wildfire (WF) injuries, measured as percentage (\%) of P-selectin positive platelets (A) or P-selectin median fluorescence intensity (MFI) (B) at rest and in the presence of $1 \mu \mathrm{M}$ arachidonic acid by flow cytometry. Arachidonic acid (AA) caused a significant decrease in percentage of P-selectin positive platelets $(P<0.0001)$ with minimal effects on P-selectin MFI $(B)$. 

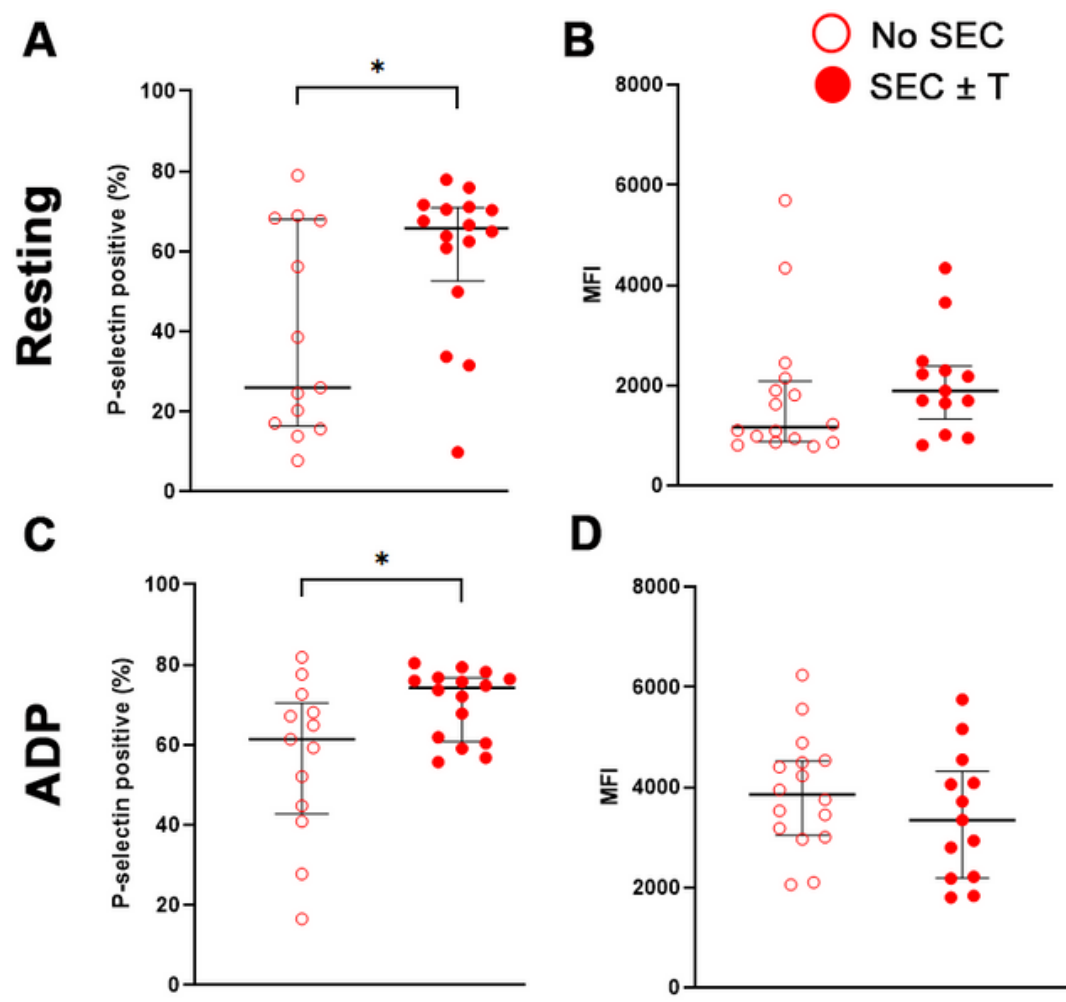

D
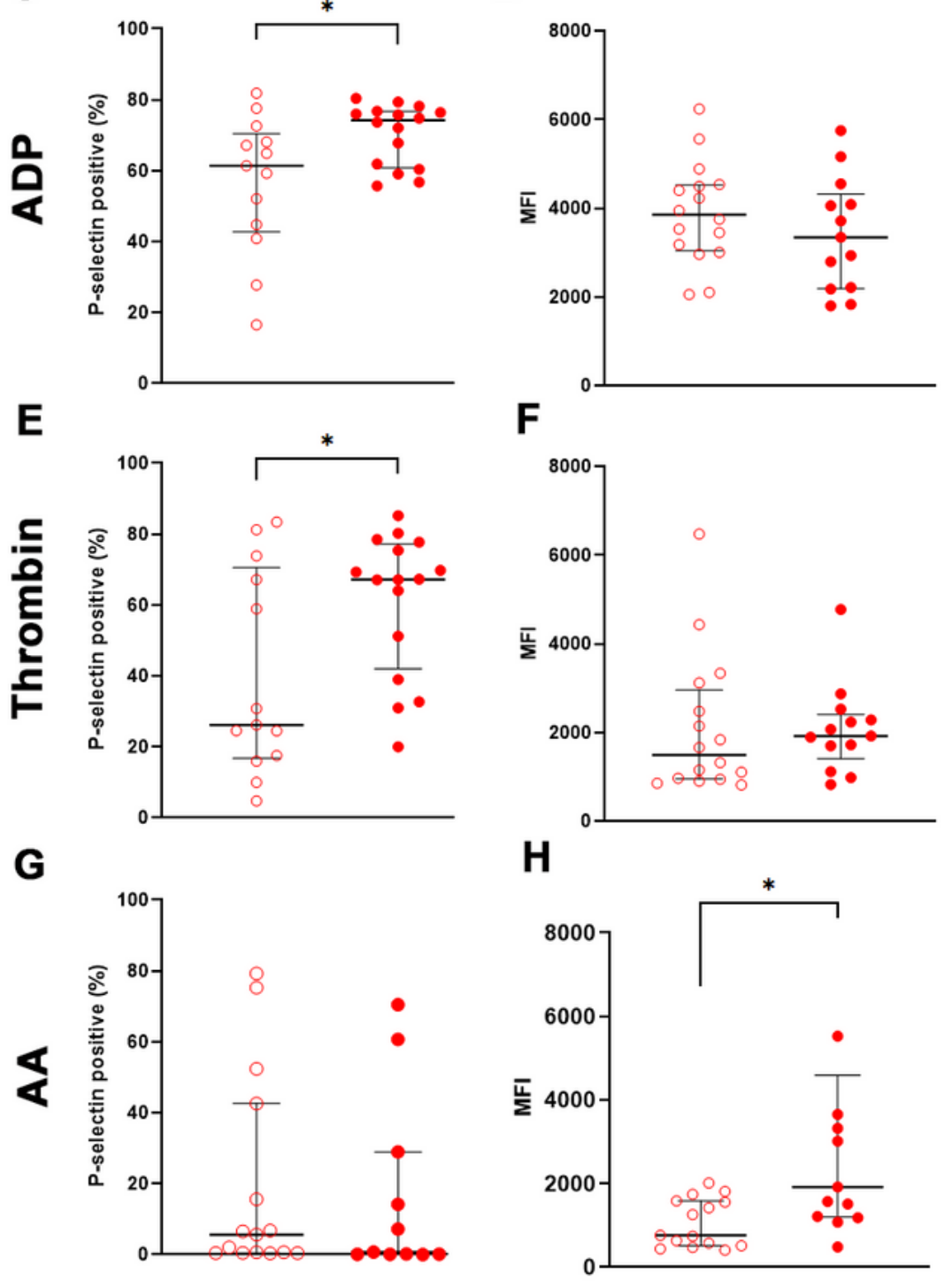

\section{Figure 4}

Cats with naturally occurring wildfire (WF) injuries had increased activated platelets with platelet priming. Platelet activation in 29 cats with or without spontaneous echocardiographic contrast \pm cardiac thrombi $(\mathrm{SEC} \pm \mathrm{T}$ ) was measured as percentage of P-selectin-positive platelets and P-selectin median florescence intensity (MFI) at rest (A-B), or in the presence of ADP (C-D), thrombin (E-F) or arachidonic acid (AA) (G-H). (A) Cats with SEC $\pm \mathrm{T}$ had increased amount of circulating activated platelets. There was also evidence 
of increased platelet priming in cats with SEC $\pm T$ once platelets were activated. (C,E) Higher percentages of P-selectin positive platelets were found in cats with SEC $\pm T$ following treatment of platelets with ADP or thrombin. $(\mathrm{H})$ Of the 3 agonists, only arachidonic acid $(\mathrm{AA})$ was able to upregulate P-selectin MFI in cats with $\mathrm{SEC} \pm \mathrm{T}$. * denotes $\mathrm{P}<0.05$.
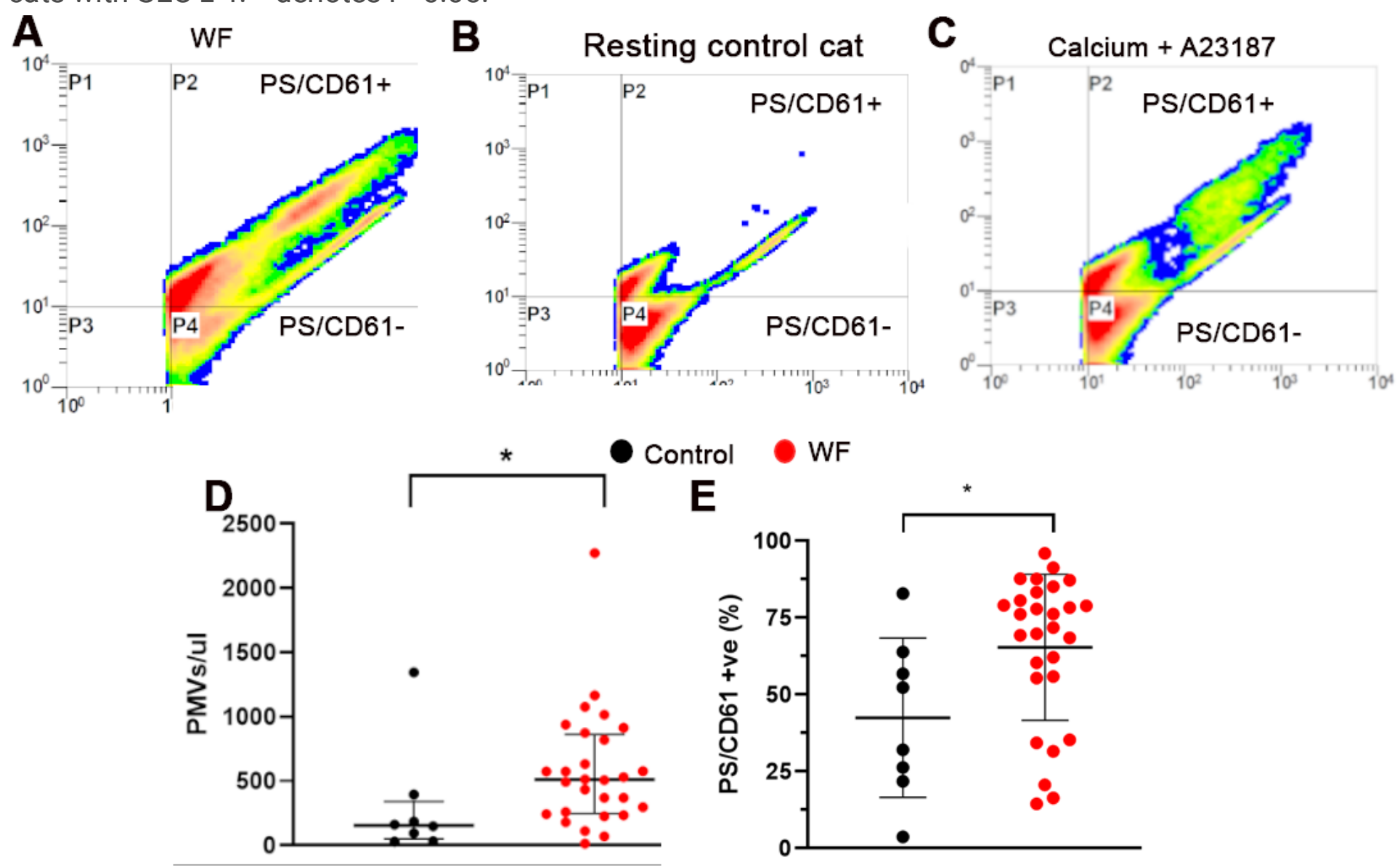

\section{Figure 5}

Cats with naturally occurring wildfire (WF) injuries had elevated circulating platelet-derived microvesicles (PDMV). PDMV was measured in platelet poor plasma in $29 \mathrm{WF}$ cats and 8 healthy control cats by flow cytometry. (A-C) Representative scatter dot plot diagrams of flow cytometric analysis of PDMV identified based on size, side scatter properties, as well as, co-expression of integrin $\beta 3$ (CD61) and phosphatidylserine (PS) - P2. (B) Representative scatter plot generated from a healthy cat without hypertrophic cardiomyopathy. (C) Positive control, included by activating platelets using calcium and the calcium ionophore, A23187, showed a significant increase in PDMV compared to resting sample. WF cats not only had increased number of PDMV but a greater percentage of them had externalized PS compared to healthy controls. * denotes $\mathrm{P}<0.05$ 